\title{
Myocardial Fiber Length Mapping with MR Diffusion Tensor Imaging
}

\author{
Y. Wu ${ }^{1},{ }^{*}$ E.X. Wu ${ }^{1,2,3}$, K.K. Wong ${ }^{1,3}$, H. Tang ${ }^{4}$, H.F. Tse ${ }^{5}$, C.P. Lau ${ }^{5}$, E.S. Yang ${ }^{1,3}$ \\ Department of Electrical and Electronic Engineering ${ }^{1}$, Medical Engineering Program ${ }^{2}$, The Jockey Club MRI \\ Centre $^{3}$, Department of Medicine ${ }^{5}$, The University of Hong Kong, Hong Kong SAR, China \\ Dept. of Radiology ${ }^{4}$, Columbia University, New York, USA \\ "Corresponding author: ewu@eee.hku.hk
}

\begin{abstract}
Diffusion tensor MRI is emerging as a rapid, nondestructive method to map myocardial fiber organization. A precise biological description of myocardial fiber performance requires knowledge of four variables: length, force, velocity and time. However, study of quantification of myocardial fiber length is lacking. The current study aims to show myocardial fiber length maps of formalin-fixed heats. Diffusion tensor MRI with medium diffusion resolution (15 directions) was performed in one isolated pig heart. Fiber length maps were investigated in multiple short-axis slices. The results provide supplementary information of myocardial fiber organization. To our knowledge, the present study is the first report of the myocardial fiber length mapping.
\end{abstract}

\section{INTRODUCTION}

Diffusion tensor MRI is a new means to elucidate the microscopic anatomical characteristics of the myocardium. Numerous studies have been reported, most of which focus on fiber orientation [1-5]. Typically, more axially oriented fibers are found near the epicardium and endocardium and in the papillary muscles. Mid-wall fibers run predominately in the circumferential direction. Results are always validated with traditional histological methods. Similarly, several studies on fiber shortening have been proposed, some of which use MR imaging tagging [6-8], and some of which use strain measurements obtained by means of in conjunction with fiber orientations [1,9]. Results show that principal thickening is predominantly radially oriented throughout the myocardium in cardiac deformation. Whereas fiber shortening is uniform, radial wall thickening increase form base to apex. However, the mechanical performance of the myocardium depends upon factors intrinsic to the contractility and upon the physicochemical conditions surrounding the cells. A precise description of cardiac muscle performance requires knowledge of fiber length [10]. In the present study, the term "myocardial fiber" designates a tissue bundle of interconnected cardiomyocytes embedded in an extracellular matrix. But research in fiber length has been lacking. In this paper, LV myocardium fiber length is investigated in an isolated healthy pig heart using diffusion tensor MRI. The aim is to obtain fiber length distribution and show supplementary information about myocardial fiber structure.

\section{METHODS}

\section{A. MR Imaging}

Imaging experiments were conducted on a 3T Philips Achieva MR imager with a quasar gradient (Philips Medical Systems; Best; Netherlands). The formalin-fixed pig heart samples were suspended in a cylinder and placed inside an 8-channel SENSE Head coil. After the determination of the LV long axis, the LV short axis planes were prescribed to cover the whole heart. Diffusion Tensor (DT) imaging was used with spin echo, echo planar imaging (SE-EPI). Imaging parameters were the following: echo time, 45ms; repetition time, $4.0 \mathrm{~s}$; field of view, 130x $130 \mathrm{~mm}^{2}$; slice thickness, 3mm; slice gap, $0 \mathrm{~mm}$; diffusion sensitivity of b, $800 \mathrm{~s} / \mathrm{mm}^{2}$; gradient direction, 15; and number of averages, 23. Images were acquired with a $128 x$ 128 data matrix. These parameters yielded a resolution of 1 $\times 1 \times 3 \mathrm{~mm}^{3}$. A total of 22 short axis images were acquired with total scan time $\sim 30 \mathrm{~min}$. Multiple averaging was employed to maximize the SNR in the image data so to minimize the interference of noise in fiber tracking calculations.

\section{B. Fiber Tracking}

The image registration and three-dimensional reconstruction of fiber tracks were obtained by using the PRIDE software package (Philips Medical Systems; Best; Netherlands). One representative slice located in middle ventricle (the $9^{\text {th }}$ slice, closer to the base, out of total 22 slices) was shown in Fig. 1. Direct RGB -> XYZ mapping scheme was used for visualization of fractional anisotropy map of LV myocardium, in which the elements oriented along the $\mathrm{X}$ axis (left/right) are colored red, along the $\mathrm{Y}$ axis (superior/posterior) green, and $\mathrm{Z}$ axis (anterior/posterior) blue. Fibers, colored with green, orientated largely in longitudinal ( $\mathrm{Y}$ axis) are found near the epicardium, endocardium, and in the papillary muscles; whereas in the mid-wall fibers, colored with red or blue, run predominantly in the circumferential (X-Z plane) direction. These results are consistent with those of previous studies [1-5].

Termination criteria of fiber tracking should be considered. The most intuitive termination criterion is the extent of anisotropy [11]. Since fractional anisotropy (FA) indicates the fraction of the diffusion, it can be chosen as one of termination criteria. Another important criterion is the angle change between pixels [11], namely directional threshold. The directional threshold depends on the particular trajectories of tracts of interest and the image resolution. Previous study indicates that that an image with resolution of $1-3 \mathrm{~mm}$ is high enough to smoothly reconstruct the curvature of trajectories of major tracts in most tissues and the angles between connected vectors are small [11], so the termination criteria is dominated by the 


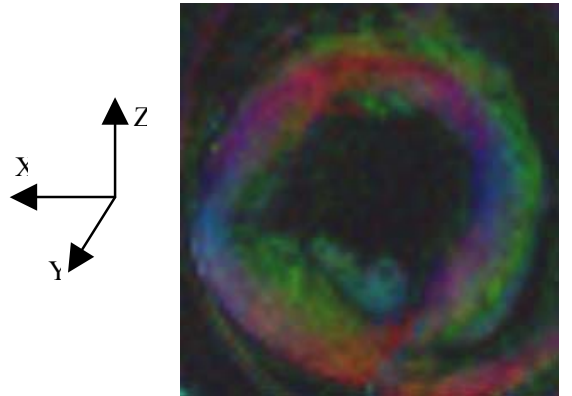

Fig. 1. FA direction map of $9^{\text {th }}$ slice closer to base.

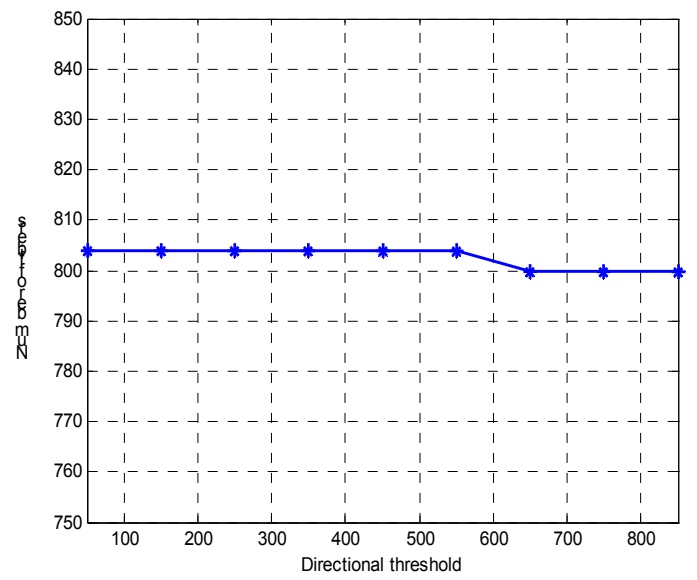

Fig. 2. Number of tibers of the $9^{\mathrm{wt}}$ slice under different directional threshold ( 0 means full deflection, 1000 means no

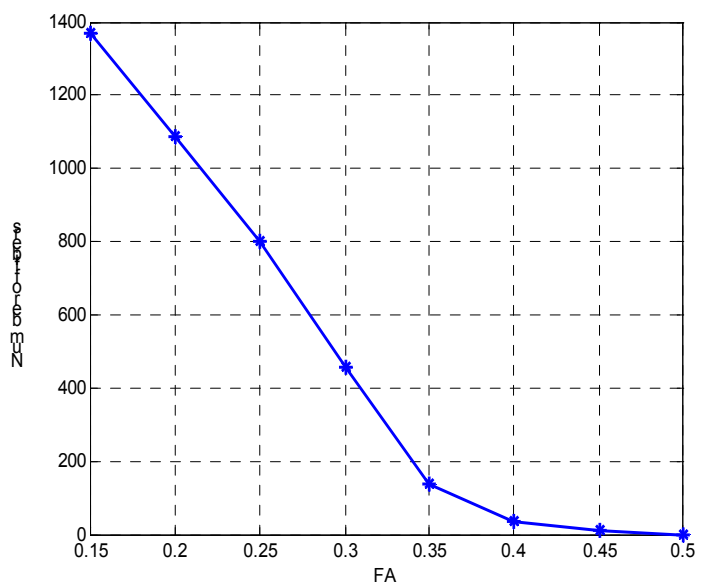

Fig. 3. Number of fibers of the $9^{\text {th }}$ slice for different FA threshold

magnitude of FA. The diffusion tensor images obtained in this study have a resolution of $1 \times 1 \times 3 \mathrm{~mm}^{3}$, thus the effect of directional threshold on tracking is likely small. Fig.2 shows the number of fibers tracked in the slice shown in Fig. 1 verse different directional thresholds for fixed FA. It demonstrates that directional thresholding indeed has little influence on fiber tracking results. Therefore, FA is the most important factor to be concerned in this case.
The effect of varying the FA thresholds on the tracking results is shown in Fig.3 and Fig.4. The number of fibers decreases with increasing of FA threshold. For relatively high $\mathrm{FA}$, for example $\mathrm{FA}=0.5$, no tracking result was obtained (Fig.3) or myocardium fibers were not continuous (Fig.4 (a)). If a lower FA threshold used, for example $\mathrm{FA}=0.15$, more marginal regions of the fiber tract were included in the tracking results (Fig.4 (d)). Similar results are seen in other slices.
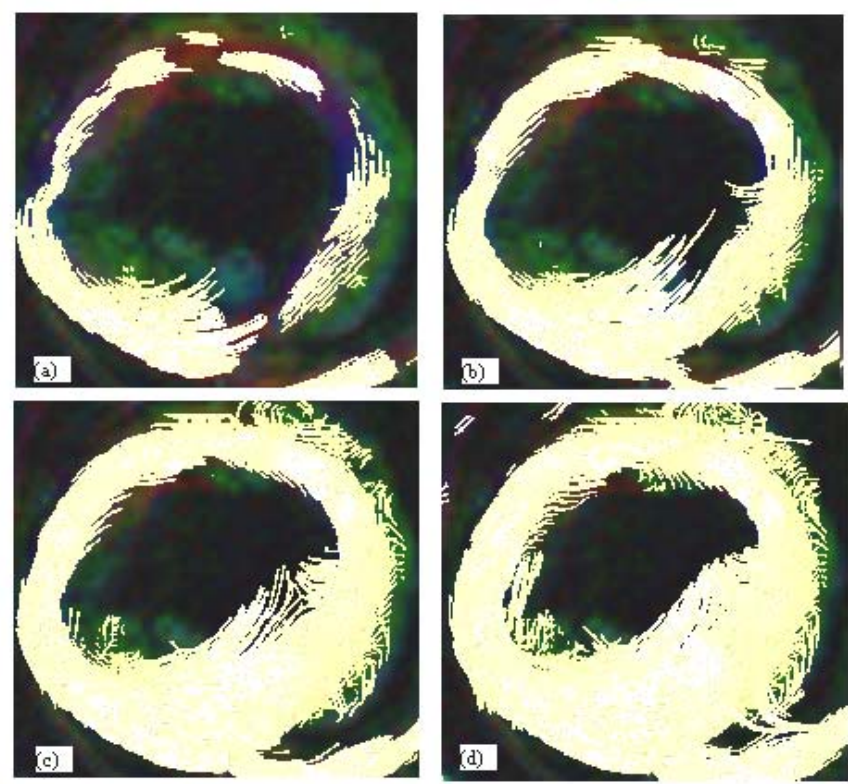

Fig. 4. Fiber tracking of the $9^{\text {th }}$ slice under FA threshold of (a) 0.30, myocardium fibers discontinuous (b) 0.25 (c) 0.2 (d) 0.15 , marginal regions of the fiber tract included

\section{Myocardial Fiber Length Mapping}

Multiple short axis slices covering the entire left ventricular myocardium were examined. Length of fibers was calculated pixel by pixel on each slice. Such fiber length calculations were also performed with varying FA thresholding values. Note that fibers going between left ventricular and right ventricular myocardium were included in the calculations. For each slice, approximately $80 \times 80$ pixel matrix was used to cover the left ventricular region.

\section{RESULTS}

Eight representative short-axis fiber length maps (slice 7 to 14) are shown in Fig. 5, together with corresponding FA direction and magnitude maps. FA threshold of 0.15 was used for the fiber tracking and length calculations. Distinct patterns are observed in the fiber length maps, indicating variations of fiber length along both the transmural and radial directions at different slice locations. Myocardial fibers crossing the slices located at middle and upper positions are observed to have relatively long fiber lengths. 

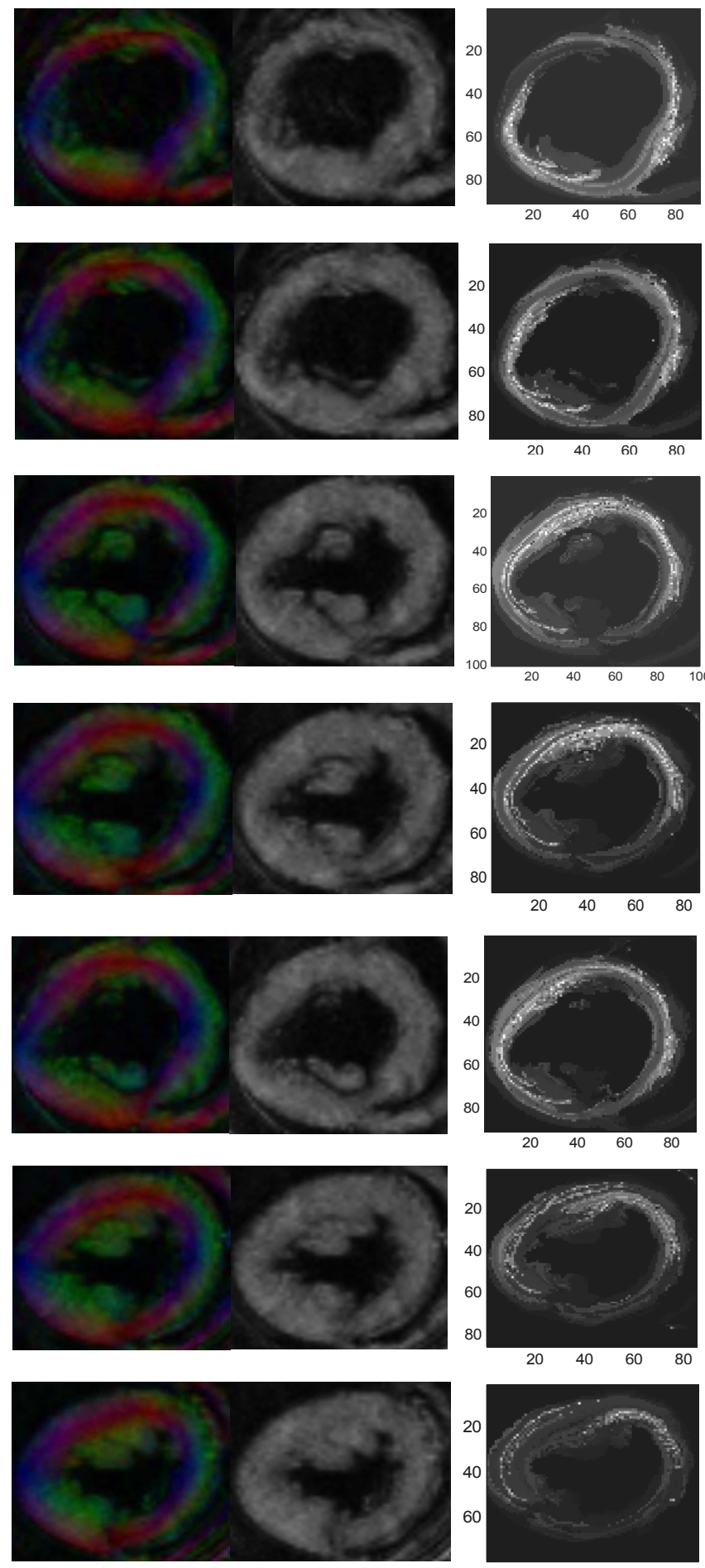

$20 \quad 40 \quad 60$
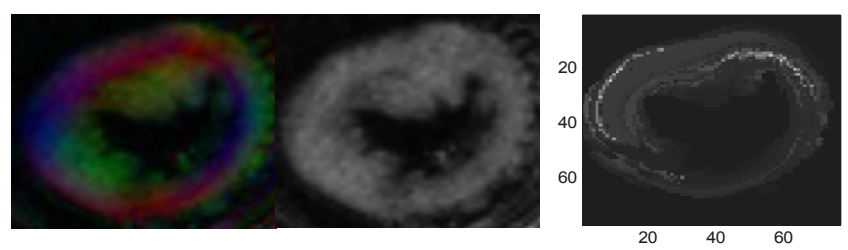

Fig. 5. FA direction maps (left), FA magnitude maps (middle), and computed fiber length maps (right) at 8 contiguous short axis locations. FA threshold of 0.15 was used for the fiber tracking and length calculation. Slice 7 to Slice 14 are from top to bottom.

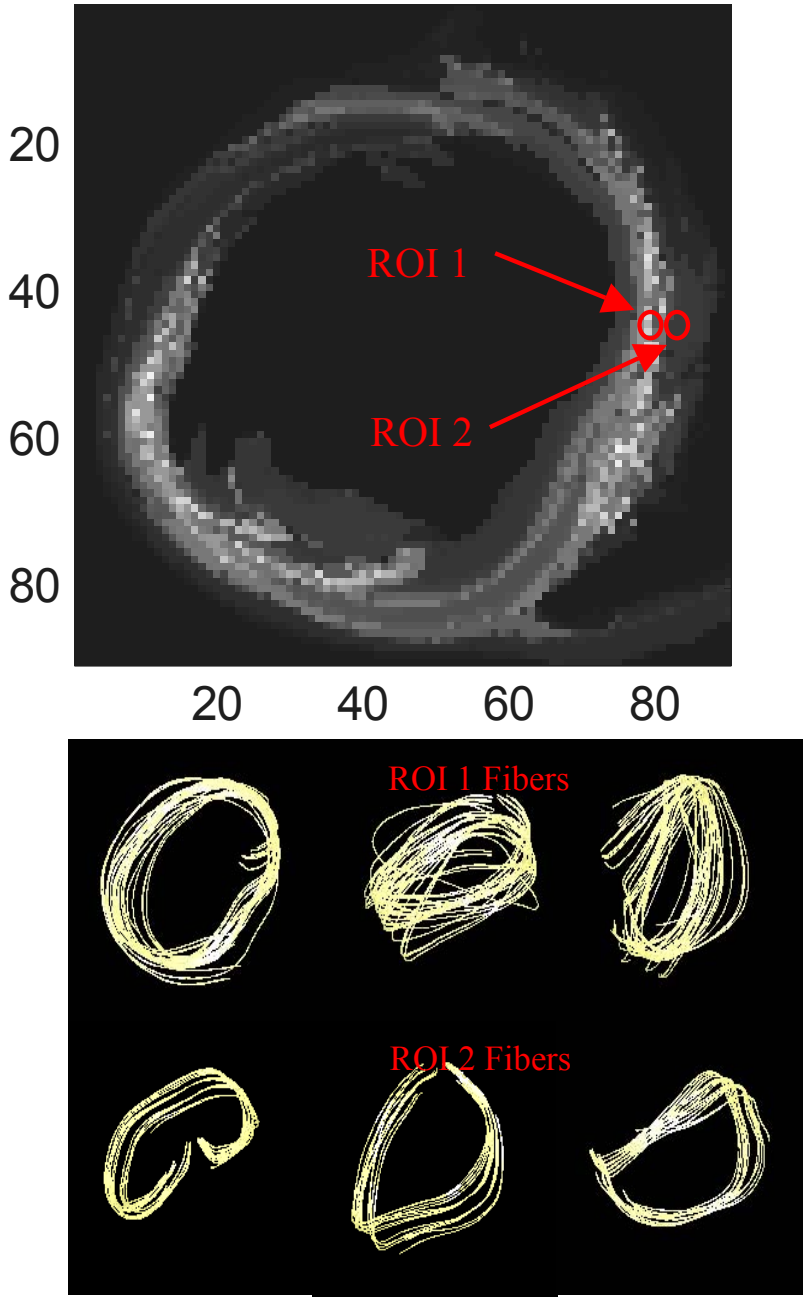

Fig. 6. Myocardial fiber bundles going through two small ROIs located on the fiber length map (slice 7). Note that two ROIs are adjacent to each but have dramatically different fiber lengths. Three views are provided to visualize the fiber bundles, coronal (left), transverse (middle), and sagittal (right). Note these two myocardial fiber bundles run in very different directions.

Fig. 6 illustrates the orientation and length information of two fiber bundles that run through two adjacent ROIs in the fiber length map (slice 7). These two ROIs have dramatically different fiber length values while the fiber bundles have different orientations and paths. These results highlight the multiple sheets of myocardial fiber bundles with different orientations and length at different transmural locations.

The FA threshold value employed in fiber tracking can influence the calculation of fiber numbers and lengths. Fiber length maps are computed for various FA threshold values. Fig. 7 shows the fiber length map with FA threshold of $0.15,0.18$, and 200. Reduced FA threshold relaxes the fiber tracking and leads to increase 
in computed fiber numbers and lengths as shown in Fig. 4. However, the pattern of the fiber length map is largely unaltered, as shown in Fig. 7.

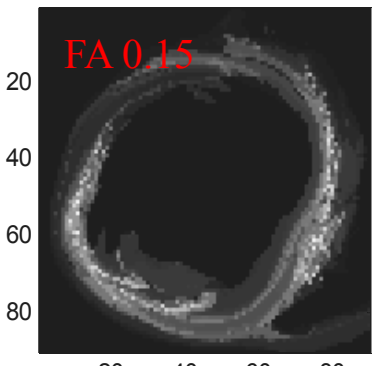

$20 \quad 40 \quad 60 \quad 80$

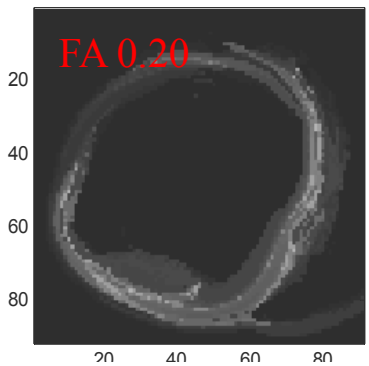

Fig. 7. Fiber length maps (slice 7) computed with different FA threshold values.

\section{DISCUSSION AND CONCLUSION}

In summary, the myocardial fiber length mapping was proposed and demonstrated in fixed normal pig heart samples based using MR diffusion tensor imaging. The acquired information is highly complementary to these from the fiber orientation studies previously reported by others, e.g., in visualizing the myocardial fiber bundles and sheets. More quantitative analysis of fiber length maps together with fiber orientations may lead to better understanding of the complex myocardial mechanics during the cardiac contractions. More data analysis is underway.

\section{ACKNOWLEDGEMENT}

The authors acknowledge the Animal Lab of the Department of Medicine, The University of Hong Kong, for preparing pig heart samples. This work was in part supported by the Hong Kong Jockey Club Charities Trust and HKU Seed Funding.

\section{REFERENCES} Myocardial fiber shortening in humans: initial results of MR imaging. Radiology, vol. 216, pp. 128- 139, 2000

[2] L. Geerts, P. Bovendeerd, K. Nicolay, T. Arts. Characterization of the normal cardiac myofiber field in goat measured with MR-diffusion tensor imaging. AJP - heart, vol. 283, pp. 139- 145, 2002

[3] E.W. Hsu, A. L. Muzikant, S. A. Matulevicius, R.C. Penland, C.S. Henriquez. Magnetic resonance myocardial fiber-orientation mapping with direct histological correlation. Am. J. Physiol, Vol 274, pp. H1627- H1634, 1998

[4] Y. Jiang, K. Pandya, O. Smithies, E.W. Hsu. Three-dimensional diffusion tensor microscopy of fixed mouse hearts. Magn Reson Med, vol. 52, pp. 453- 460, 2004

[5] A.A. Holmes, D.F. Scollan, R.L. Winslow. Direct histological validation of diffusion tensor MRI in formaldehyde-fixed myocardium. Magn Reson Med, vol. 44, pp. 157- 161, 2000

[6] C.H. Scott, M.S.J. Sutton, N. Gusani, Z. Fayad, D. Kraitchman, et al. Effect of dobutamine on regional left ventricular function measured by tagged magnetic resonance imaging in normal subjects. Am j Cardiol, vol. 83, pp. 412-417, 1999

[7] J. Garot, D.A. Bluemke, N.F. Osman, C.E. Rochitte, E.R. McVeigh, et al. Fast determination of regional myocardial strain fields from tagged cardiac images using harmonic phase MRI. Circulation, vol. 101, pp. 981-988, 2000

[8] S.B. Yeon, N. Reichek, B.A. Tallant, J.A.C. Lima, L.P. Calhoun, et al. Validation of in vivo myocardial strain measurement by magnetic resonance tagging with sonomicrometry. Journal of American College of Cardiology, vol. 38, pp. 555-561, 2001

[9] K.D. Costa, Y. Takayama, A.D. McCulloch, J.W. Covell. Laminar fiber architecture and three-dimensional systolic mechanics in canine ventricular myocardium. Am. J. Physiol, vol.276, pp. H595-H607, 1999

[10] H. Pouleur, M.F. Rousseau, A.A. Charlier. Limitations of the clinical assessment of myocardial contractility. Schweiz Med Wochenschr, vol. 115, pp. 638-643, May 1985

[11] S. Mori, P.C.M. van Zijl. Fiber tracking: principle and strategiesa technical review. NMR Biomed, vol.15, pp. 468-480, 2002 